\title{
Der Digital Twin leidet nicht
}

\section{Marc Melchert}

Dr. med., Facharzt für Psychiatrie und Psychotherapie, Mitglied FMH

Die junge Ärztin kratzt sich am Kopf und schaut verlegen im Raum hin und her. Sie nimmt ihren ganzen Mut zusammen, schaut dem Patienten direkt in die Augen und sagt diesem: «Es ist mir sehr peinlich, aber

"Ich habe keinen Zugang zu Ihrem Digital Twin und weiss nicht, wie es Ihnen heute geht.»

wie Sie in den Nachrichten gehört haben sind unsere Datensysteme zusammengebrochen, und wir haben zurzeit keinen Zugang zu den DHD, weil alle CRS abgeschaltet wurden. Die Verarbeiter und Besitzer der medizinischen Daten haben den zentralen Server für den Zugriff gesperrt. Aus Sicherheitsgründen erfolgte in allen Spitälern eine Notabschaltung, es wurde das gesamte System heruntergefahren. Ich habe keinen Zugang zu Ihrem Digital Twin und habe deshalb keine Ahnung, was Ihnen fehlt und wie es Ihnen heute geht. Ich muss jetzt etwas sehr Unangenehmes machen, das habe ich in meiner Laufbahn als Ärztin noch nie machen müssen.»
Betretenes Schweigen, lange Pause, dann rafft sich die junge Ärztin zusammen. "Ich frage Sie jetzt ganz direkt und offen: 'Wie geht es Ihnen?'» Der alte Mann auf dem Patientenstuhl lächelt und sagt: «Schämen Sie sich nicht, als ich in ihrem Alter war, haben wir uns solche Fragen ganz offen am Morgen beim Rapport unter Kollegen gestellt, manchmal sogar schon in der S-Bahn auf dem Weg zur Arbeit.» Erschrocken schaut ihn die Ärztin an und fragt: "In der S-Bahn, miteinander gesprochen? - und sogar so persönliche Fragen?» Der alte Mann antwortet: «Ja, so war das, wir sprachen noch darüber, wie es uns geht. Als ich Arzt

«Ein weiterer Algorithmus berechnet den Befindlichkeits-Koeffizienten.»

war, fragten wir sogar unsere Patienten solche Fragen: 'Wie geht es Ihnen?', 'Wie fühlen Sie sich heute?', und in Privatkliniken wurde sogar gefragt: 'Was bedeutet diese Krankheit für Sie?'» Die junge Ärztin ist erstarrt, schaut den älteren Kollegen fassungslos an

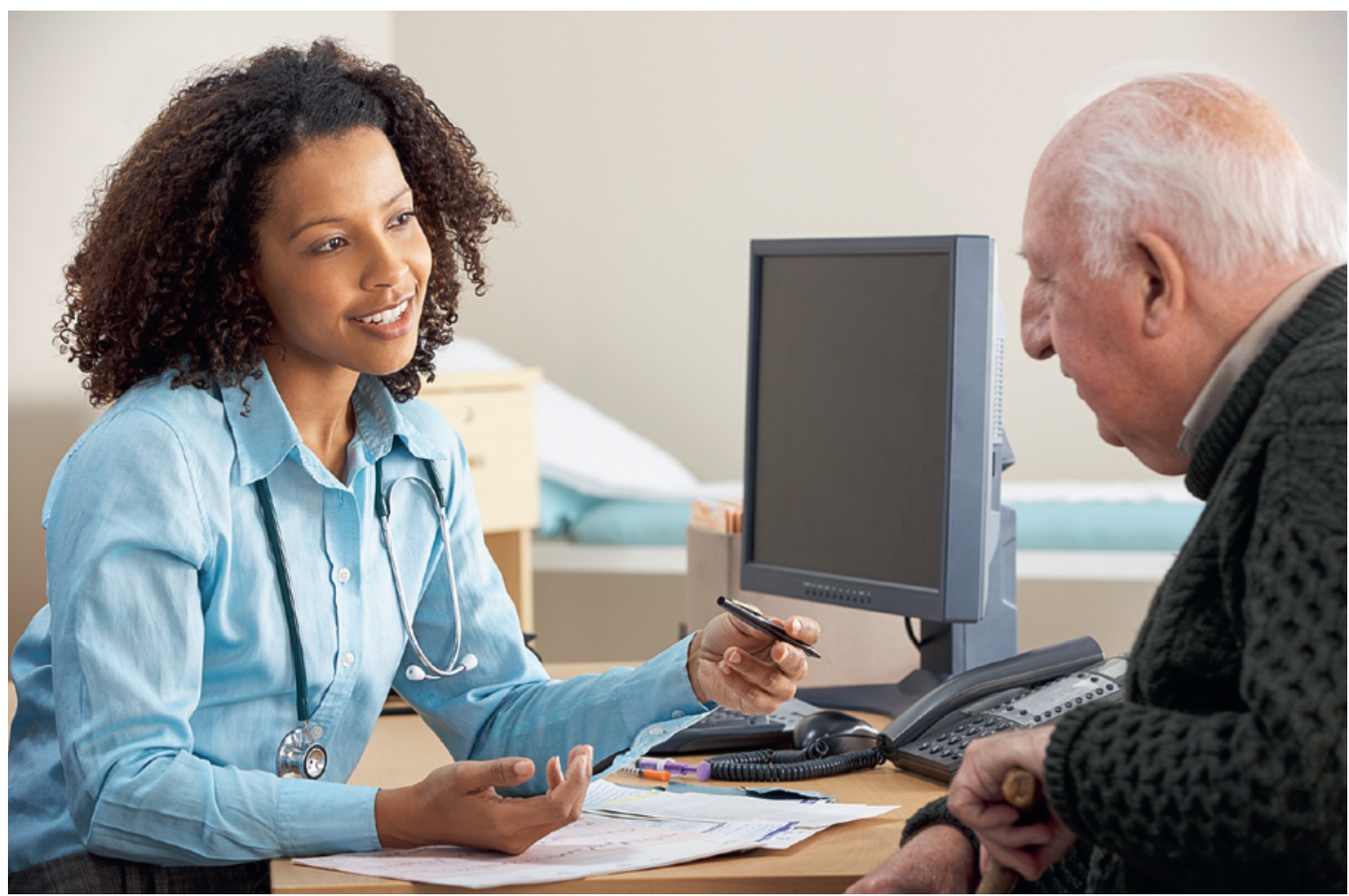

Patientengespräch (Symbolbild). 
und sagt laut und aufgeregt: «Aber wussten die Patienten darauf eine Antwort?»

\section{«Die Patienten haben verlernt, über sich und ihr Leiden zu berichten.»}

Das DHD (Digital Health Data) und die CRS (Card-Reader-Station) sind seit einigen Jahren in Betrieb genommen worden, und niemand kann sich vorstellen, wie es früher ohne gewesen war. Alle haben ein DHD-Armband, darauf werden die Daten gespeichert, die den Träger betreffen: persönliche Daten, Versicherungsnummer und die Gesundheitsdaten. So ist auf dem Armband ein Digital Twin (eine digitale Repräsentanz des Armbandträgers) gespeichert. Revolutionär ist auch, dass die stündlich gemessenen Daten wie Puls, Pulsvariabilität, Blutdruck und Sauerstoffsättigung mittels Algorithmen den vegetativen Zustand des Patienten errechnen können. Aus diesen und weiteren Daten errechnet ein weiterer Algorithmus den BF (Befindlichkeits-Koeffizienten). Im Eingang jeder Arztpraxis, jedes Spitals, Krankenwagens sind die CRS-Lesegeräte, welche die Daten aus dem Armband ablesen und auf einen Bildschirm übertragen.

\section{«Empathie war schon damals keine Tarif- position.»}

Als die Ärztin später beim Krisenrapport mit der Spitalleitung über die seltsame Begegnung mit dem alten Patienten spricht, werden alle nachdenklich, und einer wagt es dann auszusprechen: «Solche Leute brauchen wir jetzt! Seit die Systeme DHD und CRS lahmgelegt wurden, wissen wir nichts mehr über die Patienten, und kaum jemand von uns hat gelernt, wie man an solche Informationen kommt. Seit es Telefone mit Nummernspeicher gibt, können wir die Telefonnummern nicht mehr auswendig; als die Navigationsgeräte kamen, haben wir das Kartenlesen verlernt, und jetzt mit dem System der Digital Twins haben wir verlernt, mit den Patienten zu reden. Das Schlimmste daran ist, dass die Patienten verlernt haben, über sich und ihre Beschwerden zu berichten! Wer ist dieser Mann?» Die junge Ärztin fährt fort: «Er war früher Arzt und hat mir im Vertrauen gestanden, früher hätten sie in gewissen Fällen sogar eine ganze Stunde mit einem Patienten gesprochen.» Ein Raunen geht durch die Menge, einige schütteln ungläubig den Kopf, einer sagt mürrisch: «Über was soll man eine ganze Stunde lang reden können!»

Der alte Arzt kenne noch ein paar Leute, die diese Kunst immer noch als Hobby übten. Sie haben alle zwei Monate eine Zusammenkunft und sprechen miteinander. Er behauptet, dass an diesen Abenden die Teilnehmer sogar ihre Telefone abstellen und ohne Geräte direkt miteinander kommunizieren. Einige von denen sollen sogar noch die Handschrift beherrschen. Misstrauisch schauen alle die junge Ärztin an, und der mürrische Kollege platzt heraus: «Alte Männer, die Handschrift können und miteinander reden, das tönt nach Freimaurerei!» Der Chefarzt steht auf und sagt energisch: «Keine Polemik, wir sind auf jede Lösung angewiesen, lassen wir den Kollegen rein, er soll uns berichten. Wir sind auf jeden Lösungsansatz angewiesen!»

Der alte Arzt kommt rein und erzählt von der Zeit, als man mit den Patienten sprach: über die Symptome, die seelische Befindlichkeit und manchmal sogar über die Stimmung. Er erzählt auch davon, dass die Patienten sich selber und ihre Symptome ganz gut beschreiben konnten. Durch die Gespräche und die vielen Fragen waren sie geübt, sich selber zu beobachten und auch über ihr Leiden zu berichten. Der alte Mann macht eine Runde, fragt jeden am runden Tisch: «Wie geht es Ihnen, was bedeutet dieser Shut-down für Sie selber, was geben Sie diesem Geschehen für eine persönliche Bedeutung?» Es entsteht eine aufgeregte kreative Stimmung. Nach einer guten Stunde platzt der Chefarzt heraus: «Das ist sensationell, seit einer Stunde sprechen wir alle miteinander, ohne uns gegenseitig lahmzulegen, und ich höre kreative Ideen für das Krisenmanagement. Diese Leute sollen uns schulen.»

\section{«Nostalgie zu einer Zeit, als die Beziehungs- qualität noch einen Wert hatte.»}

"Ich werde an der nächsten Krisensitzung aller Chefärzte einen Antrag stellen für ein Budget, wir brauchen diese Ausbildung.» Der alte Arzt schaut auf und sagt: «Es gibt tatsächlich einige von unserer Gruppe, die können das noch, ich werde diese zusammentrommeln! Eines sei aber am Schluss erwähnt: Es wird kein grosses Budget gebraucht. Wir arbeiten aus Überzeugung und aus Nostalgie zu einer Zeit, als Medizin eher heilen (medere) als messen (metire) bedeutete. Schon damals gab es für Empathie keine Taxpunkte, und die Beziehungsqualität war nicht Pflicht, sondern Kür.» Lange Pause und betretenes Schweigen im Raum. Der Skeptiker am Tisch faucht leise vor sich hin: "Ich habe ja gewarnt, da ist etwas faul an der Sache: Freimaurer, Sekte - oder noch schlimmer Hausärzte und Psychiater, man munkelt, diese hätten sich früher schon für sowas interessiert.»

Bildnachweis

다 Monkey Business Images | Dreamstime.com 